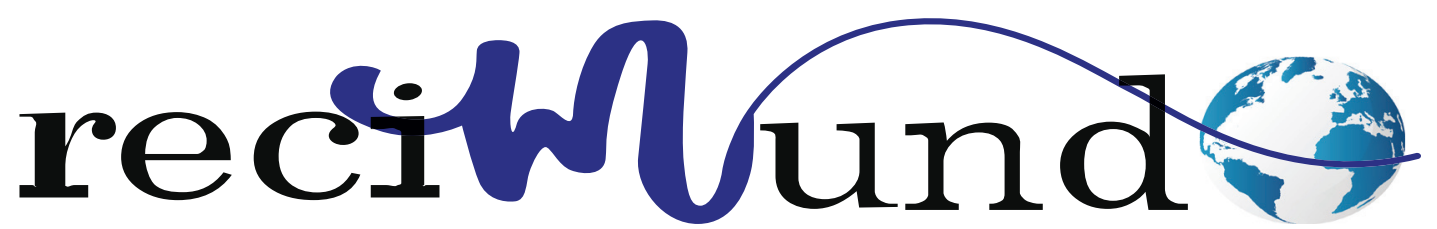

Revista Científica Mundo de la Investigación y el Conocimiento

DOI: 10.26820/recimundo/4.(4).octubre.2020.496-508

URL: http://recimundo.com/index.php/es/article/view/974

EDITORIAL: Saberes del Conocimiento

REVISTA: RECIMUNDO

ISSN: 2588-073X

TIPO DE INVESTIGACIÓN: Artículo de Revisión

CóDIGO UNESCO: 32 Ciencias Médicas

PAGINAS: 496-508

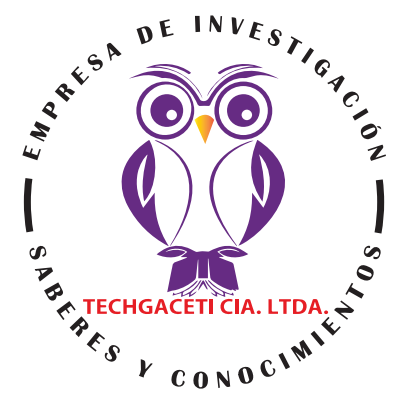

\title{
Causas y efectos de los pacientes diagnosticados con el mal de Parkinson
}

Causes and effects of patients diagnosed with Parkinson's disease

Causas e efeitos dos doentes diagnosticados com a doença de Parkinson Jeniffer Maricruz Muentes Solorzano; Johanna Katherine Lucas Quijijie'; Tania del Rocío Menéndez Pin33; Jonathan Vinicio Cornejo Vera ${ }^{4}$

\section{RECIBIDO: 10/11/2020 ACEPTADO: 26/11/2020 PUBLICADO: 13/12/2020}

1. Médico Cirujano; Hospital General Manta IEES; Ecuador; jmmuentes@hotmail.com; iD https://orcid.org/0000-0003-37683864

2. Médico Cirujano; Médico General en Funciones Hospitalarias; IESS Manta; Ecuador; kattysan1125@hotmail.com; (iD https://orcid.org/0000-0001-9572-8050

3. Licenciada en Enfermería; Doctorante de Salud Pública; Universidad Técnica de Manabí; Portoviejo, Ecuador; tania.menendez@utm.edu.ec; iD https://orcid.org/0000-0002-3646-0100

4. Médico Cirujano; Médico Residente del Área de Cirugía en Hospital Dr. Rafael Rodríguez Zambrano; Manta, Ecuador; jonathan26jg@hotmail.com; (iD) https://orcid.org/0000-0002-3708-8355

CORRESPONDENCIA

Jeniffer Maricruz Muentes Solorzano

jmmuentes@hotmail.com

Manta; Ecuador

○ RECIMUNDO; Editorial Saberes del Conocimiento, 2020 


\section{RESUMEN}

La enfermedad de Parkinson (EP) es un trastorno neurológico progresivo conocido por una gran cantidad de características motoras y no motoras que pueden afectar la función en un grado variable. Esta revisión describe las características clínicas de la EP con énfasis en aquellas que diferencian la enfermedad de otros trastornos parkinsonianos. Debido a que no existe una prueba definitiva para el diagnóstico de la EP, la enfermedad debe diagnosticarse con base en criterios clínicos. El temblor en reposo, la bradicinesia, la rigidez y la pérdida de los reflejos posturales se consideran generalmente los signos cardinales de la EP. La presencia y la presentación específica de estas características se utilizan para diferenciar la EP de los trastornos parkinsonianos relacionados. Otras características clínicas incluyen síntomas motores secundarios (por ejemplo: Hipomimia, disartria, disfagia, sialorrea, micrografía, marcha arrastrando los pies, festinación, congelación, distonía, reflejos glabelares), síntomas no motores (por ejemplo: Disfunción autonómica, anomalías cognitivas / neuroconductuales, trastornos del sueño y anomalías sensoriales como anosmia, parestesias y dolor). La ausencia de temblor en reposo, la aparición temprana de dificultad para caminar, la inestabilidad postural, la demencia, las alucinaciones y la presencia de disautonomía, oftalmoparesia, ataxia y otras características atípicas, junto con una respuesta deficiente o nula a la levodopa, sugieren diagnósticos distintos de la EP. Un conocimiento profundo del amplio espectro de manifestaciones clínicas de la EP es fundamental para el diagnóstico adecuado de la enfermedad. Las mutaciones o variantes genéticas, las anomalías en la neuroimagen y otras pruebas son biomarcadores potenciales que pueden mejorar el diagnóstico y permitir la identificación de personas en riesgo.

Palabras clave: Enfermedad de Parkinson, Temblor, Trastorno neurologico.

\section{ABSTRACT}

Parkinson's disease (PD) is a progressive neurological disorder characterized by a large number of motor and non-motor characteristics that can affect function to a variable degree. This review describes the clinical characteristics of PD with emphasis on those characteristics that differentiate the disease from other parkinsonian disorders. Because there is no definitive test for the diagnosis of PD, the disease must be diagnosed based on clinical criteria. Tremor at rest, bradykinesia, rigidity, and loss of postural reflexes are generally considered the cardinal signs of PD. The presence and specific presentation of these features are used to differentiate PD from related parkinsonian disorders. Other clinical features include secondary motor symptoms (eg, hypomimia, dysarthria, dysphagia, hypersalivation, micrograph, shuffling gait, feast, frostbite, dystonia, glabellar reflexes), non-motor symptoms (eg, autonomic dysfunction, cognitive / neurobehavioral abnormalities, sleep disturbances, and sensory abnormalities such as anosmia, paresthesia, and pain). The absence of tremor at rest, the early onset of difficulty walking, postural instability, dementia, hallucinations, and the presence of dysautonomia, ophthalmoparesis, ataxia, and other atypical features, along with a poor or no response to levodopa, suggest diagnoses other than PD. A thorough understanding of the broad spectrum of clinical manifestations of PD is essential for the proper diagnosis of the disease. Genetic mutations or variants, neuroimaging abnormalities, and other tests are potential biomarkers that can improve diagnosis and allow identification of people at risk.

Keywords: Parkinson's disease, Tremor, Neurological disorder.

\section{RESUMO}

A doença de Parkinson (DP) é uma perturbação neurológica progressiva caracterizada por um grande número de características motoras e não motoras que podem afectar a função num grau variável. Esta revisão descreve as características clínicas da DP com ênfase nas características que diferenciam a doença de outras doenças de Parkinson. Como não existe um teste definitivo para o diagnóstico da DP, a doença deve ser diagnosticada com base em critérios clínicos. Tremor em repouso, bradicinesia, rigidez, e perda dos reflexos posturais são geralmente considerados os sinais cardinais da DP. A presença e a apresentação específica destas características são utilizadas para diferenciar a DP das perturbações parkinsonianas relacionadas. Outras características clínicas incluem sintomas motores secundários (por exemplo, hipomimia, disartria, disfagia, hipersalivação, micrografia, marcha embaralhada, festa, congelação, distonia, reflexos glabelares), sintomas não motores (por exemplo, disfunção autonómica, anomalias cognitivas / neuro-comportamentais, distúrbios do sono, e anomalias sensoriais tais como anosmia, parestesia, e dor). A ausência de tremor em repouso, o início precoce da dificuldade de marcha, instabilidade postural, demência, alucinações, e a presença de disautonomia, oftalmoparesia, ataxia, e outras características atípicas, juntamente com uma resposta fraca ou nenhuma resposta à levodopa, sugerem outros diagnósticos para além da DP. Uma compreensão profunda do amplo espectro de manifestações clínicas da DP é essencial para o diagnóstico adequado da doença. Mutações ou variantes genéticas, anomalias neuro-imaginosas e outros testes são biomarcadores potenciais que podem melhorar o diagnóstico e permitir a identificação de pessoas em risco.

Palavras-chave: Doença de Parkinson, Tremor, Desordem neurológica. 


\section{Introducción}

El Parkinson es una enfermedad común del trastorno neurodegenerativo con síntomas motores y no motores. La evidencia reciente sugiere que la degeneración del Parkinson comienza en el núcleo motor dorsal del vago nervio y núcleo olfatorio, seguido por el tronco cerebral inferior, luego el basal ganglios y prosencéfalo y se extiende hacia la corteza. "Esta patología difusa significa que tanto dopaminérgicos como no dopaminérgicos las vías neuronales se ven afectadas, lo que resulta en numerosas discapacidades motoras y no motoras" (Allen \& Moloney, 2015).

Las deficiencias no motoras son amplias e incluyen deficiencias cognitivas y emocionales, disfunción autonómicas, trastornos del sueño y anomalías sensoriales, como dolor. Muchas personas con la enfermedad de Parkinson tiene dolor crónico y, según se informa, lo experimenta más del $80 \%$ de esta población. "El dolor ha sido calificado como uno de los síntomas más molestos, y se asocia con una reducción de la calidad de vida relacionada con la salud y una mayor ansiedad y depresión" (Ozturk \& Kocer, 2016).

Entrando un poco en historia se tiene, en su ensayo sobre la parálisis temblorosa" Parkinson, (2002) describió por primera vez "el síndrome clínico que más tarde llevaría su nombre". Identificó seis casos, tres de los cuales examinó personalmente; estos casos fueron observados en las calles de Londres. Anteriormente conocida como "parálisis agitante", Charcot más tarde en el siglo XIX dio crédito al Parkinson al referirse a la enfermedad como "enfermedad de Parkinson" o enfermedad de Parkinson (EP).

Charcot también reconoció las formas no trémulos de EP y señaló correctamente que la lentitud de movimiento debe distinguirse de la debilidad o "disminución de la potencia muscular", un término utilizado originalmente por Parkinson. Pasaron más de 100 años (1919) después de la descripción original de Parkinson antes de que se reconociera que los pacientes con EP pierden células en la sustancia negra, y pasaron 140 años (1957) antes de que Carlsson y compañia descubrieran la dopamina como un neurotransmisor putativo en Lund, Suecia (Bjorklund \& Dunnett, 2007).

El descubrimiento de Birkmayer \& Hornykiewicz, (1961) de que "las concentraciones de dopamina están marcadamente disminuidas en el cuerpo estriado de los pacientes con EP" conllevó al camino para los primeros ensayos de levodopa en pacientes con EP, el año siguiente dando cabida a la posterior concesión del Premio Nobel de Medicina a Carlsson en 2000. "La capacidad de la levodopa inyectada para mejorar la acinesia en pacientes con EP se demostró por primera vez en 1961 y fue seguida por el desarrollo de levodopa oral más adelante en la década" (Birkmayer \& Hornykiewicz, 1961).

Más recientemente, mutaciones genéticas, manejo anormal de proteínas mal plegadas por los sistemas ubiquitina-proteasoma y autofagia-lisosomal, aumento del estrés oxidativo, disfunción mitocondrial, inflamación y otros mecanismos patogénicos han sido identificados como factores contribuyentes en la muerte de dopaminérgicos y células no dopaminérgicas en el cerebro de pacientes con EP. Este artículo se centra en las causas, efectos, características clínicas de la EP y la diferenciación de la enfermedad de otros trastornos parkinsonianos con el fin de brindar información que pueda servir para el desarrollo de futuras investigaciones.

\section{Metodología}

Para el desarrollo de este proceso investigativo, se plantea como metodología la encaminada hacia una orientación científica particular que se encuentra determinada por la necesidad de indagar en forma preci- 
sa y coherente una situación, en tal sentido Davila, (2015) define la metodología "como aquellos pasos anteriores que son seleccionados por el investigador para lograr resultados favorables que le ayuden a plantear nuevas ideas" (p.66)

Lo citado por el autor, lleva a entender que el desarrollo de la acción investigativa busca simplemente coordinar acciones enmarcadas en una revisión bibliográfica con el fin de complementar ideas previas relacionadas Causas y efectos de los pacientes diagnosticados con el mal de Parkinson a través de una revisión de literatura, para así finalmente elaborar un cuerpo de consideraciones generales que ayuden a ampliar el interés propuesto.

\section{Tipo de Investigación}

Dentro de toda práctica investigativa, se precisan acciones de carácter metodológico mediante las cuales se logra conocer y proyectar los eventos posibles que la determinan. En este sentido, la presente investigación corresponde al tipo documental, definido por Castro (2016), "se ocupa del estudio de problemas planteados a nivel teórico, la información requerida para abordarlos se encuentra básicamente en materiales impresos, audiovisuales y / o electrónicos". (p.41).

En consideración a esta definición, la orientación metodológica incluye la oportunidad de cumplir con una serie de actividades inherentes a la revisión y lectura de diversos documentos, donde se encuentran ideas explicitas relacionadas con los tópicos encargados de identificar una característica inmersa en el estudio. Por lo tanto, se realizaron continuas interpretaciones con el claro propósito de revisar aquellas apreciaciones propuestas por diferentes investigadores en relación al tema de interés, para luego dar la respectiva argumentación a los planteamientos, en función a las necesidades encontradas en la investigación, apoya- dos en las herramientas tecnológicas para la búsqueda de trabajos con valor científico disponibles en la web que tenían conexión con el objetivo principal de la investigación.

\section{Fuentes Documentales}

El análisis correspondiente a las características que predomina en el tema seleccionado, llevan a incluir diferentes fuentes documentales encargadas de darle el respectivo valor científico y en ese sentido cumplir con la valoración de los hechos a fin de generar nuevos criterios que sirven de referencia a otros procesos investigativos. Para Castro,(2016) las fuentes documentales incorporadas en la investigación documental o bibliográfica, "representa la suma de materiales sistemáticos que son revisados en forma rigurosa y profunda para llegar a un análisis del fenómeno" (p.41). Por lo tanto, se procedió a cumplir con la lectura previa determinada para encontrar aquellos aspectos estrechamente vinculados con el tema, con el fin de explicar mediante un desarrollo las respectivas apreciaciones generales de importancia.

\section{Técnicas para la Recolección de la Infor- mación}

La conducción de la investigación para ser realizada en función a las particularidades que determinan a los estudios documentales, tiene como fin el desarrollo de un conjunto de acciones encargadas de llevar a la selección de técnicas estrechamente vinculadas con las características del estudio. Bolívar, (2015), refiere, que es "una técnica particular para aportar ayuda a los procedimientos de selección de las ideas primarias y secundarias". (p.71).

Tal como lo expresa, Bolívar, (2015) "Las técnicas documentales proporcionan las herramientas esenciales y determinantes para responder a los objetivos formulados y llegar a resultados efectivos" (p. 58). Es decir, para responder con eficiencia a las

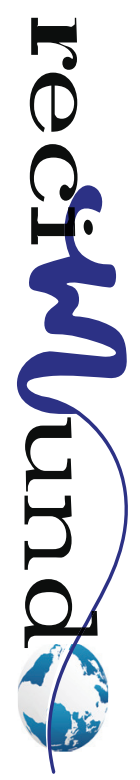


necesidades investigativas, se introdujeron como técnica de recolección el método inductivo, que hizo posible llevar a cabo una valoración de los hechos de forma particular para llegar a la explicación desde una visión general. El autor Bolívar, (2015) tambien expresa que las técnicas de procesamiento de datos en los estudios documentales "son las encargadas de ofrecer al investigador la visión o pasos que deben cumplir durante su ejercicio, cada una de ellas debe estar en correspondencia con el nivel a emplear" (p. 123). Esto indica, que para llevar a cabo el procesamiento de los datos obtenidos una vez aplicadas las técnicas seleccionadas, tales como: fichas de resumen, textual, registros descriptivos entre otros, los mismos se deben ajustar al nivel que ha sido seleccionado.

\section{Resultados}

\section{Características clínicas}

Hay cuatro características cardinales de la EP que pueden agruparse bajo el acrónimo TRAP (Por sus siglas en ingles): temblor en reposo, rigidez, acinesia (o bradicinesia) e inestabilidad postural. Además, la postura flexionada y la congelación (bloqueos motores) se han incluido entre las características clásicas del parkinsonismo, siendo la EP la forma más común. Debido a los diversos perfiles y estilos de vida de los afectados por la EP, las discapacidades motoras y no motoras deben evaluarse en el contexto de las necesidades y objetivos de cada paciente.

Se utilizan varias escalas de calificación para la evaluación de la discapacidad motora y la discapacidad en pacientes con EP, pero la mayoría de estas escalas no se han evaluado completamente en cuanto a validez y confiabilidad.

La escala de Hoehn y Yahr se usa comúnmente para comparar grupos de pacientes y para proporcionar una evaluación general de la progresión de la enfermedad, que va desde la etapa 0 (sin signos de enfermedad) hasta la etapa 5 (en silla de ruedas o en cama a menos que reciba asistencia). La escala de calificación unificada de la enfermedad de Parkinson (UPDRS) es la escala mejor establecida para evaluar la discapacidad y el deterioro (Ebersbach, Baas, \& Csoti, 2006).

Los estudios que utilizan UPDRS para rastrear la progresión de la EP sugieren que el curso de la EP no es lineal y que la tasa de deterioro es variable y más rápida en la fase temprana de la enfermedad y en pacientes con inestabilidad postural y dificultad para caminar (PIGD) de PD. En un "estudio prospectivo de 145 pacientes clínicos seguidos durante 1 año y de 124 pacientes comunitarios seguidos durante 4 años, la tasa media anual de deterioro en las puntuaciones motoras y de discapacidad osciló entre el $2,4 \%$ y el $7,4 \%$ " (Schrag, Dodel, \& Spottke, 2007).

La UPDRS actual está siendo revisada para que la escala sea más sensible al detectar pequeños cambios e integre elementos no motores. Otros tipos de escalas de calificación incluyen las que evalúan las manifestaciones psiquiátricas por ejemplo, la depresión y la calidad de vida. Las características clínicas más frecuentes asociadas con la EP se enumeran en la figura 1 y se comentan en las siguientes secciones. 


\section{ENFERMEDAD DE PARKINSON \\ Se presenta fundamentalmente con síntomas motores y suele aparecer entre los 60 y 70 años}

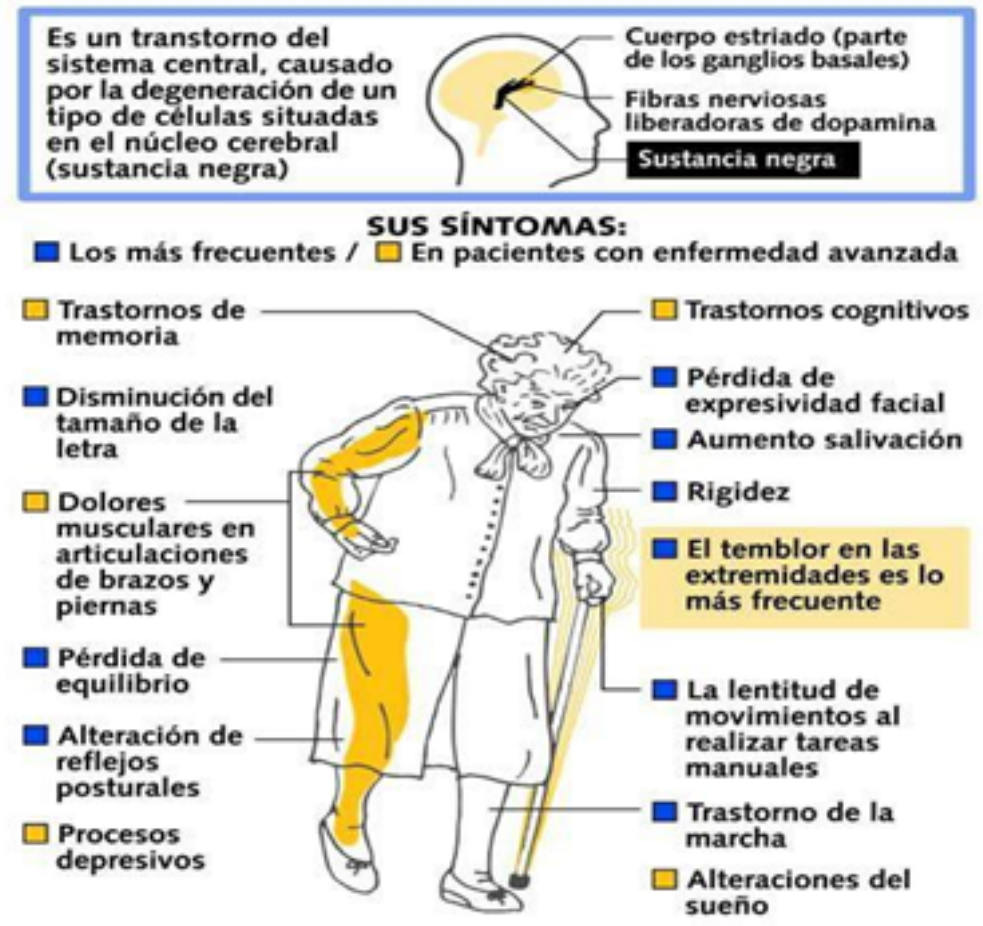

Figura 1. Síntomas de la enfermedad de Parkinson

Fuente: (Schrag, Dodel, \& Spottke, 2007)

\section{Bradicinesia}

La bradicinesia se refiere a la lentitud del movimiento y es el rasgo clínico más característico de la EP, aunque también se puede observar en otros trastornos, incluida la depresión. "La bradicinesia es un sello distintivo de los trastornos de los ganglios basales y presenta dificultades para planificar, iniciar y ejecutar el movimiento y para realizar tareas secuenciales y simultáneas" (Parkinson Federacion Española , 2017) .

La manifestación inicial suele ser lentitud en la realización de las actividades de la vida diaria y lentitud en los tiempos de movimiento y reacción.

Esto puede incluir dificultades con tareas que requieren control de la motricidad fina (por ejemplo, abrocharse, usar utensilios). Otras manifestaciones de bradiquinesia in- cluyen la pérdida de movimientos espontáneos y gestos, babeo debido a la deglución deteriorada, disartria monotónica y hipofonica, pérdida de expresión facial (hipomimia) y la disminución de parpadear, y la reducción de balanceo de los brazos al caminar. (Ozturk \& Kocer, 2016)

Dado que la bradicinesia es uno de los síntomas más fácilmente reconocibles de la EP, puede ser evidente antes de cualquier examen neurológico formal. La evaluación de la bradicinesia generalmente incluye que los pacientes realicen movimientos rápidos, repetitivos y alternos de la mano (golpecitos con los dedos, agarre, pronación-supinación de la mano) y golpeteo del talón y observar no solo lentitud sino también disminución de la amplitud.

Aunque la fisiopatología de la bradicinesia no ha sido bien delineada, "es la caracterís- 
tica cardinal de la EP la que parece correlacionarse mejor con el grado de deficiencia de dopamina" (Juri \& Vivian, 2016). Esto está respaldado por la observación de una disminución de la densidad neuronal en la sustancia negra en pacientes ancianos con parkinsonismo independientemente del diagnóstico de EP. "Además, la tomografía por emisión de positrones en pacientes con PD ha demostrado que la disminución de la absorción deF-fluorodopa en el cuerpo estriado y accumbens-caudado complejo es proporcional al grado de bradicinesia" (Lozza, Marie, \& Baron, 2002).

Se plantea la hipótesis de que la bradicinesia es el resultado de una alteración de la actividad normal de la corteza motora mediada por una función dopaminérgica reducida. "Los estudios de neuroimagen funcional también sugieren un deterioro en el reclutamiento de los sistemas corticales y subcorticales que regulan los parámetros cinemáticos del movimiento (p. Ej., La velocidad)" (Juri \& Vivian, 2016). A la inversa, el reclutamiento de diversas áreas premotora, tales como los responsables del control visuomotor, se incrementa.

Anatómicamente, el déficit parece estar localizado en el putamen y el globo pálido, resultando en una reducción de la fuerza muscular producida al inicio del movimiento. "El análisis de las grabaciones electromiográficas mostró que los pacientes con bradicinesia son incapaces de energizar los músculos apropiados para proporcionar suficiente fuerza para iniciar y mantener grandes movimientos rápidos" (Turner, Grafton, \& Mclntosh, 2003). Debido a que los pacientes con EP tienen una actividad electromiográfica disminuida, necesitan una serie de ráfagas de agonistas múltiples para lograr movimientos más grandes.

\section{Temblor}

El temblor en reposo es el síntoma más común y fácilmente reconocible de la EP.
Los temblores son unilaterales, ocurren con una frecuencia entre 4 y $6 \mathrm{~Hz}$ y casi siempre son prominentes en la parte distal de una extremidad. Los temblores de las manos se describen como temblores de supinación-pronación ("rodar la píldora") que se propagan de una mano a la otra. El temblor en reposo en pacientes con EP también puede afectar los labios, el mentón, la mandíbula y las piernas pero, a diferencia del temblor esencial, rara vez afecta el cuello / cabeza o la voz. (Fernández, 2015)

Por tanto, un paciente que se presenta con temblor de cabeza probablemente tenga temblor esencial, distonía cervical o ambos, en lugar de EP. Característicamente, el temblor en reposo desaparece con la acción y durante el sueño. Algunos pacientes también informan un temblor "interno" que no está asociado con un temblor visible. El temblor de la EP se diferencia del temblor esencial por una serie de características.

Algunos pacientes con EP tienen antecedentes de temblor postural, fenomenológicamente idéntico al temblor esencial, durante muchos años o décadas antes del inicio del temblor parkinsoniano u otras características relacionadas con la EP. Los autores (Shahed \& Jankovic, 2007) "han proporcionado un creciente cuerpo de evidencia que indica que el temblor esencial es un factor de riesgo para la EP".

Además del temblor en reposo, muchos pacientes con EP también tienen temblor postural que es más prominente e incapacitante que el temblor en reposo y puede ser la primera manifestación de la enfermedad. "El temblor postural relacionado con el Parkinson ("temblor reemergente") se diferencia del temblor esencial en que la aparición del temblor a menudo se retrasa después de que el paciente asume una posición horizontal estirada" (Fernández, 2015). Dado que el temblor reemergente ocurre con la misma frecuencia que el temblor de reposo clásico y responde a la terapia dopaminér- 
gica, es probable que represente una variante del temblor de reposo más típico.

De acuerdo con Fernández, (2015)

Hay varias pistas para el diagnóstico de temblor esencial existente cuando coexiste con la EP, que incluyen un historial prolongado de temblor de acción, antecedentes familiares de temblor, temblor de cabeza y voz, y ausencia de latencia cuando los brazos están extendidos en una posición horizontal frente al cuerpo, aunque algunos pacientes también pueden tener un temblor reemergente relacionado con su EP, caligrafía y espiral temblorosa, y mejoría del temblor con alcohol y bloqueadores beta (p. 9).

La aparición de temblores en reposo es variable entre los pacientes y durante el curso de la enfermedad. "Los estudios clínico-patológicos han demostrado que los pacientes con EP y temblor prominente tienen degeneración de un subgrupo de neuronas del mesencéfalo (A8), mientras que esta área se conserva en pacientes con EP sin temblor" (Shahed \& Jankovic, 2007).

\section{Rigidez}

La rigidez "se caracteriza por una mayor resistencia, generalmente acompañada del fenómeno de la rueda dentada, especialmente cuando se asocia con un temblor subyacente, presente en todo el rango de movimiento pasivo de una extremidad (flexión, extensión o rotación alrededor de una articulación)" (GPC, 2016).
Puede ocurrir proximalmente (por ejemplo, cuello, hombros, caderas) y distalmente (muñecas, tobillos). "Las maniobras de refuerzo como los movimientos voluntarios de la extremidad contralateral), conocido como la maniobra de la Froment, por lo general aumentan esta característica y son particularmente útiles en la detección de los casos leves de la rigidez" (GPC, 2016). Puede asociarse con dolor, y el hombro doloroso es una de las manifestaciones iniciales más frecuentes de la EP, aunque comúnmente se diagnostica erróneamente como artritis, bursitis o lesión del manguito rotador.

\section{Deformidades posturales}

La EP puede producir rigidez del cuello y el tronco (rigidez axial), lo que da lugar a posturas axiales anormales (por ejemplo, anterocolis, escoliosis). "Las deformidades posturales que dan como resultado una postura flexionada del cuello, tronco, codos y rodillas flexionadas, a menudo se asocian con rigidez" (GPC, 2016). Sin embargo, la postura flexionada generalmente ocurre al final de la enfermedad. En algunos pacientes también se pueden desarrollar deformidades de las extremidades estriadas (por ejemplo, mano estriada, dedo del pie estriado).

La mano estriatal se caracteriza por la desviación cubital de las manos, la flexión de las articulaciones metacarpofalángicas, la extensión de la proximal y la flexión de las articulaciones interfalángicas distales (fig. 2A); El pie estriado se caracteriza por la extensión o flexión (fig. 2B) de los dedos (Ashour \& Jankovic, 2006). 


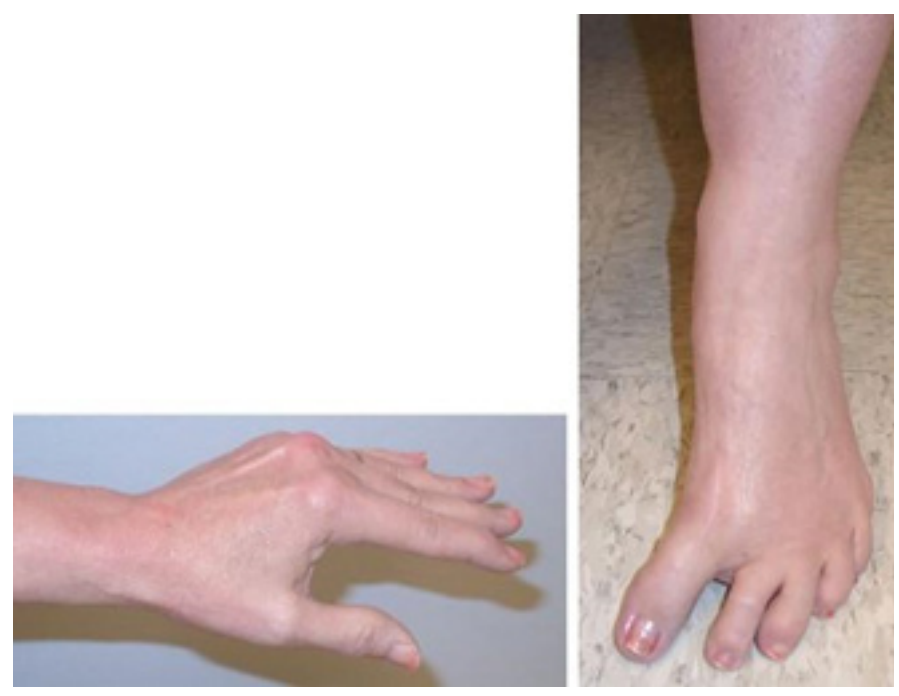

Figura 2. Deformidad estriatal de la mano $(A)$ y el pie $(B)$ en un paciente con enfermedad de Parkinson típica.

Fuente: (Schrag, Dodel, \& Spottke, 2007)

Los pacientes con deformidades estriatales tienden a ser más jóvenes y a experimentar un inicio más temprano de los síntomas parkinsonianos iniciales. Otras anomalías esqueléticas incluyen flexión extrema del cuello ("cabeza caída" o "columna doblada"), flexión del tronco (camptocormia) y escoliosis.

La camptocormia se caracteriza por una flexión extrema de la columna toracolumbar. La condición se agrava al caminar y se alivia al sentarse, acostarse en decúbito supino o al extender voluntariamente el tronco cuando el paciente se apoya contra una pared, un andador alto o una mesa (fig. 3A-C). (Williams, Watt, \& Lees, 2006)

Además de la EP, otras causas de camptocormia incluyen "distonía y miopatía del tronco extensor.Otra deformidad del tronco es el síndrome de Pisa, que se caracteriza por una inclinación del tronco, especialmente al estar sentado o de pie" (Williams, Watt, \& Lees, 2006)

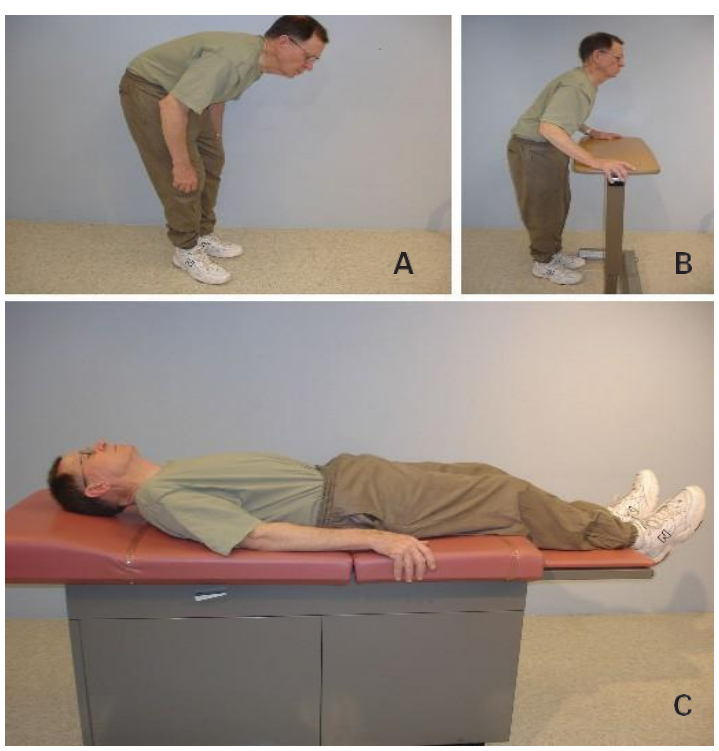

Figura 3. Camptocormia en un paciente con enfermedad de Parkinson que se manifiesta por flexión del tronco $(A)$ que el paciente puede corregir empujándose a sí mismo a la posición de extensión (B) o acostado en decúbito supino $(\mathrm{C})$.

Fuente: (Schrag, Dodel, \& Spottke, 2007) 


\section{Inestabilidad postural}

La inestabilidad postural debida a la pérdida de reflejos posturales es generalmente una manifestación de las etapas tardías de la EP y generalmente ocurre después del inicio de otras características clínicas. La prueba de tracción, en la que los hombros empujan rápidamente al paciente hacia atrás o hacia adelante, se utiliza para evaluar el grado de retropulsión o propulsión, respectivamente. Dar más de dos pasos hacia atrás o la ausencia de una respuesta postural indica una respuesta postural anormal. La inestabilidad postural (junto con la congelación de la marcha) es la causa más común de caídas y contribuye significativamente al riesgo de fracturas de cadera. La larga latencia hasta el inicio de las caídas diferencia a la EP de otros trastornos neurodegenerativos, como la parálisis supranuclear progresiva (PSP) y la atrofia multisistémica (MSA) (Pastora \& Tolosaa, 2001)

Otros factores también influyen en la aparición de inestabilidad postural en pacientes con EP. Estos incluyen otros síntomas parkinsonianos, hipotensión ortostática, cambios sensoriales relacionados con la edad y la capacidad de integrar información sensorial visual, vestibular y propioceptiva (cinestesia). El miedo a caerse puede afectar aún más el control del equilibrio en pacientes con EP.

El tratamiento (terapia dopaminérgica, palidotomía, estimulación cerebral profunda) puede mejorar algunos signos axiales pero generalmente no mejora de manera sólida la inestabilidad postural, medida por la inclinación de la plataforma y la inclinación visual. "Apuntar a otros núcleos para la estimulación cerebral profunda además del núcleo subtalámico y el globo pálido, como la zona incerta y el núcleo pedunculopontino, se está explorando como un posible tratamiento quirúrgico de la marcha y la estabilidad postural" (Giladi, McDermott, \& Fahn, 2001)

\section{Congelación}

La congelación, también conocida como bloqueos motores, es una forma de acinesia (pérdida de movimiento) y es uno de los síntomas más incapacitantes de la EP. Aunque la congelación es un rasgo característico de la EP, no ocurre de forma universal. La congelación afecta con mayor frecuencia a las piernas al caminar, pero también pueden afectar los brazos y los párpados. Por lo general, se manifiesta como una incapacidad repentina y transitoria (generalmente, $10 \mathrm{~s}$ ) para moverse. De acuerdo con Macht, Kaussner, \& Moller, (2007).

Esto puede incluir vacilación al comenzar a caminar (vacilación inicial) o una incapacidad repentina para mover los pies durante situaciones específicas (por ejemplo, girar o caminar por un pasaje estrecho, cruzar calles concurridas, acercarse a un destino). La congelación se asocia con importantes consecuencias sociales y clínicas para los pacientes. En particular, es una causa común de caídas (p. 6).

Se han descrito cinco subtipos de congelación: vacilación inicial, vacilación en el giro, vacilación en espacios reducidos, vacilación en el destino y vacilación en espacios abiertos. Los episodios son más graves en el estado OFF y se mitigan con la terapia con levodopa. Además, los pacientes suelen desarrollar trucos para superar los ataques de congelación. Esto incluye marchar al mando, pasar objetos (p. ej., un bastón, grietas en el suelo), caminar al ritmo de la música o un ritmo y cambiar el peso corporal.

Los factores de riesgo para el desarrollo de congelación incluyen "la presencia de rigidez, bradicinesia, inestabilidad postural y una mayor duración de la enfermedad. Por el contrario, el temblor al inicio de la enfermedad se asocia con un menor riesgo de congelación" (GPC, 2016). Dado que la congelación suele ocurrir más tarde en el curso de la enfermedad o no es el síntoma

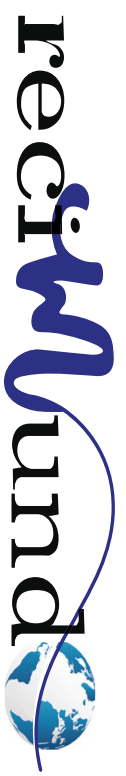


predominante, se deben considerar diagnósticos alternativos cuando ocurren estas presentaciones.

La congelación, particularmente cuando ocurre durante el período ON, no suele responder a la terapia dopaminérgica, pero se ha encontrado que los pacientes tratados con selegilina tienen un riesgo menor. "Las inyecciones de toxina botulínica, aunque eficaces para una variedad de síntomas parkinsonianos como temblores, distonía y sialorrea, no han demostrado ser consistentemente efectivas en el tratamiento de la congelación" (Singh, Pentland, \& Hunter, 2007)

Las alteraciones respiratorias en pacientes con EP pueden ser restrictivas u obstructivas. Estas complicaciones se asocian con una morbilidad y mortalidad considerables; la neumonía es un predictor independiente de mortalidad en pacientes con EP en hogares de ancianos. "El patrón obstructivo puede estar relacionado con rigidez, artrosis cervical o rango de movimiento restringido en el cuello, y el patrón restrictivo puede estar relacionado con la rigidez de la pared torácica" (GPC, 2016). La respiración también puede verse comprometida por la discinesia respiratoria relacionada con la levodopa en pacientes con EP.

\section{Evaluación de los pacientes con EP}

\section{Criterios diagnósticos}

La EP se diagnostica según criterios clínicos; no existe una prueba definitiva para el diagnóstico. "Históricamente, la confirmación patológica del característico cuerpo de Lewy en la autopsia se ha considerado el criterio estándar para el diagnóstico" (Ashour \& Jankovic, 2006). En la práctica clínica, el diagnóstico se basa típicamente en la presencia de una combinación de características motoras cardinales, síntomas asociados y excluyentes y respuesta a la levodopa.
Aunque el diagnóstico de la enfermedad de Parkinson es sencillo cuando los pacientes tienen una presentación clásica, diferenciar la enfermedad de Parkinson de otras formas de parkinsonismo puede ser un desafío temprano en el curso de la enfermedad, cuando los signos y síntomas se superponen con otros síndromes. "Los criterios de diagnóstico han sido desarrollados por el Banco de Cerebros de la Sociedad de Enfermedad de Parkinson del Reino Unido y el Instituto Nacional de Trastornos Neurológicos y Accidentes Cerebrovasculares (NINDS)" (Hughes, Ben-Shlomo, \& Daniel, 1992). Sin embargo, la confiabilidad y validez de estos criterios no se han establecido claramente.

El diagnóstico erróneo de la EP puede surgir por varias razones. Además, muchas de las características destacadas de la EP (rigidez, alteración de la marcha, bradicinesia) también pueden ocurrir como resultado del envejecimiento normal o de afecciones médicas comórbidas y multifactoriales (ejemplo: diabetes, cáncer, entre otros).

\section{Conclusiones}

Mediante el desarrollo del proceso investigativo se observó que el EP es un trastorno neurodegenerativo progresivo que se manifiesta por un amplio espectro de características motoras y no motoras. La progresión natural de la EP es variable, pero suele ser más rápida en pacientes de inicio tardío. La serie de síntomas y características son el indicativo de selección para el diagnostico de esta enfermedad.

Se asocia con una razón de mortalidad debido a las complicaciones motoras de quienes la padecen, lo que genera, riesgos de caídas y accidentes que desafortunadamente puede desencadenar en la muerte del paciente, además de las molestias y dolores que están asociadas a la enfermedad y que se incrementan con el avance. Sin embargo, debido a que no existen pruebas 
diagnósticas definitivas para el EP, los médicos requieren un conocimiento profundo de las manifestaciones clínicas para ayudar a diferenciarla de trastornos relacionados.

Las investigaciones futuras pueden proporcionar biomarcadores específicos de la enfermedad que permitan su diferenciación de otros trastornos neurodegenerativos. Dichas pruebas no solo serán útiles para diagnosticar el mal de Parkinson en las personas afectadas, sino que también serán útiles para identificar a los miembros de la familia o poblaciones en riesgo, brindando así una oportunidad para iniciar la terapia neuroprotectora en una etapa asintomática.

\section{Bibliografía}

Allen, N., \& Moloney, N. (2015). The rationale for exercise in the management of pain in Parkinson's disease. J Parkinsons Dis. , 5 (2), 229-39.

Ashour, R., \& Jankovic, J. (2006). Joint and skeletal deformities in Parkinson's disease, multiple system atrophy, and progressive supranuclear palsy. MOV Disord, 1856-63.

Birkmayer, W., \& Hornykiewicz, O. (1961). The L-3,4-dioxyphenylalanine (DOPA)-effect in Parkinson- akinesia. Wien Klin Wochenschr , 787-8.

Bjorklund, A., \& Dunnett, S. (2007). Dopamine neuron systems in the brain: an update. Trends Neurosci , 194-202.

Bolívar, J. (2015). Investigación Documental. México. Pax.

Castro, J. (2016). Técnicas Documentales. México. Limusa.

Davila, A. (2015). Diccionario de Términos Científicos. . Caracas: Editorial Oasis.

Ebersbach, G., Baas, H., \& Csoti, I. (2006). Scales in Parkinson's disease. J Neurol , 32-5.

Fernández, W. (2015). Temblor: diagnóstico diferencial y tratamiento.

Giladi, N., McDermott, M., \& Fahn, S. (2001). Freezing of gait in PD: prospective assessment in the DATATOP cohort. Neurology , 1712-21.

GPC. (2016). Diagnostico y tratamiento de la enfermedad de Parkinson. Guia de Practica Clinica.
Hughes, A., Ben-Shlomo, Y., \& Daniel, S. (1992). What features improve the accuracy of clinical diagnosis in Parkinson's disease: a clinicopathologic study. Neurology , 1142-6.

Juri, C., \& Vivian, W. (2016). NEUROIMÁGENES EN ENFERMEDAD DE PARKINSON: ROL DE LA RESONANCIA MAGNÉTICA, EL SPECT Y EL PET. Ann Neurol , 27 (3), 380-391.

Lozza, C., Marie, R., \& Baron, J. (2002). The metabolic substrates of bradykinesia and tremor in uncomplicated Parkinson's disease. Neuroimage, 688-99.

Macht, M., Kaussner, Y., \& Moller, J. (2007). Predictors of freezing in Parkinson's disease: a survey of 6,620 patients. MOV Disord , 953-6.

Ozturk, E., \& Kocer, B. (2016). Chronic pain in Parkinson's disease: Frequency, characteristics, independent factors, and relationship with health-related quality of life. J Back Musculoskelet Rehabil , 30, 101-108.

Parkinson Federacion Española . (2017). Espacio Parkinson. Retrieved Dic 30, 2020 from https://www.esparkinson.es/wp-content/ uploads/2017/11/1.2-CONOCE-LA-ENFERMEDAD-S\%C3\%ADntomas-del-p\%C3\%A1rkinson. pdf

Parkinson, J. (2002). An essay on the shaking palsy. J Neuropsychiatry Clin Neurosci, 223.

Pastora, P., \& Tolosaa, E. (2001). La enfermedad de Parkinson: diagnóstico y avances en el conocimiento de la etiología y en el tratamiento. Medicina Integral , 37 (3), 104-117.

Schrag, A., Dodel, R., \& Spottke, A. (2007). Rate of clinical progression in Parkinson's disease. A prospective study. MOV Disord, 938-45.

Shahed, J., \& Jankovic, J. (2007). Exploring the relationship between essential tremor and Parkinson's disease. Parkinsonism Relat Disord , 67-76.

Singh, R., Pentland, B., \& Hunter, J. (2007). Parkinson's disease and driving ability. J Neurol Neurosurg Psychiatry, 363-6.

Turner, R., Grafton, S., \& Mclntosh, A. (2003). The functional anatomy of parkinsonian bradykinesia. Neuroimage, 163-79.

Williams, D., Watt, H., \& Lees, A. (2006). Predictors of falls and fractures in bradykinetic rigid syndromes: a retrospective study. J Neurol Neurosurg Psychiatry , 468-73. 


\section{CITAR ESTE ARTICULO:}

Muentes Solorzano, J. M., Lucas Quijije, J. K., Menéndez Pin, T. del R., \& Cornejo Vera, J. V. (2021). Causas y efectos de los pacientes diagnosticados con el mal de Parkinson. RECIMUNDO, 4(4), 496-508. https://doi.org/10.26820/ recimundo/4.(4).octubre.2020.496-508

\section{(c)}

(1) (\$) (2)

BY NC SA

CREATIVE COMMONS RECONOCIMIENTO-NOCOMERCIAL-COMPARTIRIGUAL 4.0 . 